15

\title{
Алгоритм предварительного анализа дифракционных спектров для нанокомпозитных материалов с примесью массивной фракции
}

\author{
(C) О.А. Алексеева, ${ }^{1,2}$ А.А. Набережнов ${ }^{3}$ \\ ${ }^{1}$ Санкт-Петербургский политехнический университет Петра Великого, \\ 195251 Санкт-Петербург, Россия \\ ${ }^{2}$ Томский государственный университет систем управления и радиоэлектроники, \\ 634050 Томск, Россия \\ ${ }^{3}$ Физико-технический институт им. А.Ф. Иоффре РАН, \\ 194021 Санкт-Петербург, Россия \\ e-mail: alex.nabereznov@mail.ioffe.ru
}

Поступило в Редакцию 7 июля 2021 г.

В окончательной редакции 11 сентября 2021 г.

Принято к публикации 13 сентября 2021 г.

Рассмотрены вопросы, связанные с влиянием возможного вклада массивного материала на форму линии упругих пиков, наблюдаемых в дифракционных экспериментах по рассеянию нейтронов и/или рентгеновского излучения на нанопористых матрицах, содержащих внедренные в поры вещества. Предложен алгоритм, который позволяет получить оценку вклада от массивной фракции в дифракционные пики из анализа наблюдаемых в эксперименте искажений формы линии брэгговских пиков. Такой предварительный анализ существенно упрощает проведение полнопрофильного анализа дифрактограмм для нанокомпозитных материалов, в первую очередь для изготовленных на основе порошков молекулярных сит типа SBA-15, МСM-41, МСМ-48 и т. п.

Ключевые слова: пористые матрицы, дифракция, нанокомпозитные материалы, форма линии упругих пиков, высшие центральные моменты распределения.

DOI: 10.21883/JTF.2022.01.51866.208-21

\section{Введение}

Использование дифракционных методов является одним из самых востребованных приемов исследования нанокомпозитных материалов (НКМ), поскольку анализ дифракционных спектров позволяет получить большой объем полезной информации о структуре (и ее температурной эволюции) НКМ, об особенностях фазовых переходов в этих материалах, о внутренней организации наночастиц в порах исходных матриц, включая их размеры и анизотропию формы, и многое другое. Тем не менее в ряде случаев возникают трудности при окончательной обработке полученных данных, так как в таких НКМ, особенно приготовленных на основе молекулярных сит (типа SBA-15, MCM-41, MCM-48 и т. п.), может присутствовать примесь массивного материала, которая будет существенно искажать общую картину наблюдаемых явлений. Так, например, в работе [1] было показано, что наблюдаемый в эксперименте температурный гистерезис в поведении параметра порядка в НКМ, изготовленных на основе порошков пористых молекулярных сит 2D-SBA-15 и 3D-SBA-15, содержащих внедренный в поры сегнетоэлектрик $\mathrm{NaNO} 2$, связан не только с размерными эффектами и свойствами самой матрицы, но и с наличием примеси массивного нитрита натрия порядка (по уточненным данным, полученным методом дифференциальной сканирующей калориметрии, а также после применения изложенной в настоящей работе процедуры) около $20 \%$ от общего содержания $\mathrm{NaNO} 2$ в образце. Наличие примеси массивного материала зачастую требует проведения полномасштабного дифракционного эксперимента и полнопрофильного анализа полученных дифрактограмм, что не всегда возможно, особенно на стадии приготовления НКМ. Предлагаемый алгоритм позволяет достаточно быстро определить долю массивной фракции, причем для проведения анализа достаточно иметь один интенсивный пик. Таким образом, оказывается возможным провести предварительное тестирование НКМ, оценить качество изготовленного образца и спланировать время, необходимое для проведения полномасштабных исследований структуры (и ее температурной эволюции) данного НКМ, т.е. времени набора необходимой статистики с учетом вклада массивного материала.

\section{1. Методика проведения расчетов}

\section{1. Стартовые параметры и начальные условия}

Одним из основных исходных условий является знание инструментального разрешения используемого прибора, в первую очередь - зависимости ширины упругого пика от угла рассеяния $\theta$ падающего излучения или от межплоскостного расстояния. Эти данные, а также информация о параметрах функции, описываю- 
щей форму линии упругого пика, известны заранее и обязательно присутствуют в паспорте используемой установки. Обычно они проверяются на стандартных образцах не реже одного раза в полгода. В настоящей работе в качестве примера мы использовали установочные параметры нейтронного дифрактометра высокого разрешения FIREPOD E9 (Helmholtz Zentrum Berlin, Германия). Инструментальное уширение упругого пика $H_{\text {inst }}$ рассчитывалось по формуле

$$
H_{\text {instr }}^{2}=U \operatorname{tg}^{2} \theta+V \operatorname{tg} \theta+W
$$

где параметры $U, V, W$ были взяты из паспорта данной установки.

Предполагалось, что ширина линии рефлекса, соотвествующего массивной фазе, определяется только инструментальным разрешением, а уширение (как для массивного материала, так и для НКМ) вследствие возможных упругих напряжений отсутствует.

Ширина линии упругого пика, соотвествующего наноструктурированной фазе, рассчитывалась как суммарный вклад инструментального и размерного уширений.

В принципе увеличение ширины упругих отражений в НКМ может происходить по двум причинам: из-за размерного эффекта — в этом случае уширение $\sim 1 / \cos \theta$, и из-за наличия внутренних напряжений в наночастицах — здесь уширение пропорционально $\operatorname{tg} \theta$. В общем случае используют формулу Холла-Вильямсона [2-4]:

$$
B \cos \theta=\eta \sin \theta+k \lambda / d,
$$

где $\lambda$ - длина волны излучения в ангстремах, $B-$ уширение (ширина на полувысоте) пика, которая определяется с учетом функции, описывающей форму максимума и с учетом функции разрешения (пример см. в $[3,4]$ ), $d$ - дифракционный размер частицы, $\eta$ - величина внутренних напряжений, $2 \theta-$ положение брэгговского пика, $k$ - параметр формы, обычно он $\sim 0.9-1$. В наших расчетах мы полагали $k=1$. Из вышеизложенного хорошо видно, что вклады имеют разную зависимость от угла рассеяния и могут быть легко отделены, поэтому в дальнейшем мы ограничились только достаточно малым углом рассеяния, при котором вкладом в уширение изза внутренних напряжений можно пренебречь. В таком случае используют подход Дебая-Шеррера:

$$
H_{\text {size }}=\frac{k \lambda}{d \cos \theta}
$$

При моделировании отклика рассматривалась эволюция формы пика (100) кубической структуры с параметром элементарной ячейки $a=3.35 \AA$, длина волны падающих нейтронов составляла $\lambda=1.7982(1) \AA$. Для наноструктурированного материала в порах расчет проводился для двух значений параметра $a: 3.35 \AA$ (как для массива) и $3.345 \AA$.

\section{2. Детали расчетов}

Были рассмотрены три различных функции для описания как инструментального разрешения, так и формы линии экспериментального упругого пика: гауссиан, лоренциан и войтиан. Перекрестные случаи, когда, например, инструментальная функция описывается гауссианом, а экспериментальный спектр - лоренцианом, в настоящей работе не рассматривались. Далее рассчитывалась эволюция формы (и описывающих ее параметров) упругого пика как функция весового процента вклада от массивного материала, дифракционного размера наночастиц и приведенной выше разницы в параметре элементарной ячейки.

Суммирование вкладов из-за инструментального разрешения и размерного уширения осуществлялось по следующим формулам:

в случае гауссовского профиля линии полная ширина пика $H_{G}$ определялась как

$$
H_{G}^{2}=H_{\text {instr }}^{2}+H_{\text {size }}^{2},
$$

для лоренциана $H_{L}$

$$
H_{L}=H_{\text {instr }}+H_{\text {size }},
$$

а для расчета профиля линии, описываемого функцией Войта, являющейся по определению сверткой лоренциана и гауссиана:

$$
V(x)=L(x) \otimes G(x)=\int_{-\infty}^{+\infty} L(x-u) G(u) d u
$$

(здесь $L(x)$ и $G(x)$ - компоненты, описываемые лоренцианом и гауссианом соотвественно, с различной шириной на полувысоте $H_{L}$ и $\left.H_{G}\right)$, использовалась численная аппроксимация выражения (6) в виде линейной комбинации лоренциановского $L^{\prime}(x)$ и гауссиановского вкладов $G^{\prime}(x)$ (так называемая функция псевдо-войтиан) с одинаковой шириной на полувысоте $H_{p V}$ :

$$
p V(x)=\eta L^{\prime}(x)+(1-\eta) G^{\prime}(x)
$$

при этом параметры функции псевдовойта $p V(x) \eta$ и $H_{p V}$ рассчитывались по формулам через параметры $H_{G}$ и $H_{L}$, задаваемыми формулами (4) и (5) в соответствии с подходом, предложенным в работе [5]:

$$
\begin{gathered}
H_{p V}^{5}=H_{G}^{5}+2.69269 H_{G}^{4} H_{L}+2.42843 H_{G}^{3} H_{L}^{2} \\
+4.47163 H_{G}^{2} H_{L}^{3}+0.07842 H_{G} H_{L}^{4}+H_{L}^{5}, \\
\eta=1.36603 \frac{H_{L}}{H_{p V}}-0.47719\left(\frac{H_{L}}{H_{p V}}\right)^{2}+0.11116\left(\frac{H_{L}}{H_{p V}}\right)^{3} .
\end{gathered}
$$



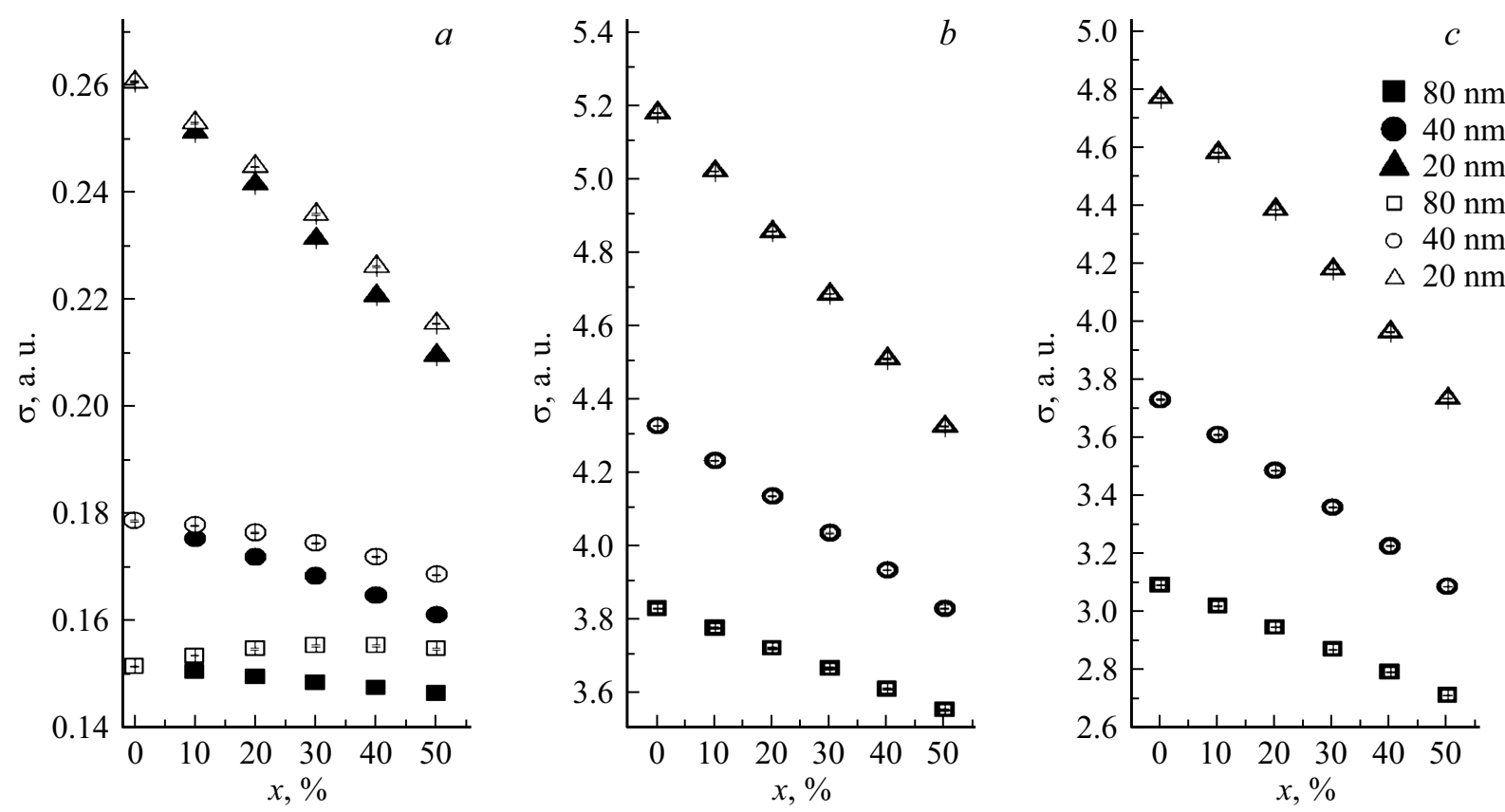

Рис. 1. Изменение дисперсии $(\sigma)$ формы линии брэгговского рефлекса при увеличении весового процентного содержания фазы массивного материала в образце $(x)$ для разных функций профиля линии (гауссиан $(a)$, лоренциан $(b)$ и псевдо-войтиан $(c)$ ), размеров частиц и разного соотношения параметров решетки массива и наноструктурированной фазы (черные символы параметры решетки одинаковы, пустые символы - параметры решетки массива и наноструктурированной фазы различаются).

На следующем этапе анализировалось поведение дисперсии $\sigma$, коэффициента асимметрии $\gamma_{1}$ и коэффициента эксцесса $\gamma_{2}$, которые рассчитывались через центральные моменты второго, третьего и четвертого порядков $\mu_{2}, \mu_{3}, \mu_{4}$ для смоделированных распределений интенсивности упругих пиков по формулам [6]:

$$
\sigma=\sqrt{\mu_{2}}, \gamma_{1}=\frac{\mu_{3}}{\sigma^{3}}, \gamma_{2}=\frac{\mu_{4}}{\sigma^{4}}-3
$$

при этом для расчета моментов для профиля линии, описываемого функциями Лоренца и Войта, для которых интегралы, определяющие моменты старших порядков, по бесконечному промежутку неопределены, интегрирование проводилось по конечному промежутку, симметричному относительно вершины пика. Полученные результаты расчетов представлены на рис. $1-4$.

\section{2. Результаты и обсуждение}

На рис. 1 представлены результаты расчетов зависимости параметра $\sigma$ от примеси массивного материала при различных указанных на рисунке размеров наночастиц для случаев гауссиана (рис. 1,a), лоренциана (рис. $1, b)$ и псевдо-войтиана (рис. $1, c)$. Отметим, что на этом рисунке и на всех последующих черные символы соответствуют случаю, когда параметры элементарной ячейки для массивной фракции и наноструктурированного материала одинаковы, кроме того, оценка ошибок расчетов на рисунках также приведена и обычно не превышает размера символа. С увеличением процентного содержания массивного материала дисперсия формы линии уменьшается, причем чем меньше размер частиц, тем сильнее это изменение. Также хорошо прослеживается зависимость от разницы в параметрах решетки $a$. В случае использования двух лоренцианов (рис. $1, b)$ и двух псевдо-войтианов (рис. $1, c$ ) для описания инструментального разрешения и экспериментального упругого пика разницы между случаями одинаковых и разных параметров решетки нет в пределах погрешности (черные и белые символы совпадают), а присутствует только зависимость $\sigma$ от примеси массивной фазы. Отметим, что положения максимумов распределений естественно зависят от разницы параметров элементарной ячейки. Из рис. 1 видно, что уже из анализа дисперсии можно сделать достаточно надежную оценку процентного вклада массивной фазы - не менее $10 \%$ - для гауссиана и лоренциана (для наночастиц с характерным размером менее $80 \mathrm{~nm}$ ) и около 5\% для псевдо-войтиана для всех исследованных размеров наночастиц.

Теперь рассмотрим поведение коэффициента эксцесса $\gamma_{2}$ от концентрации массивной фазы (рис. 2). Из рисунка хорошо видно, что этот коэффициент при увеличении содержания массивной фракции растет вне зависимости от вида профильной функции, размера частиц и соотношения параметров решетки. Для всех трех профилей наиболее сильный рост $\gamma_{2}$ соответствует менышему размеру частиц. И в этом случае можно дать достаточно надежную - не менее $10 \%$ - оценку процентного вклада массивной фазы для гауссиана (для наночастиц 

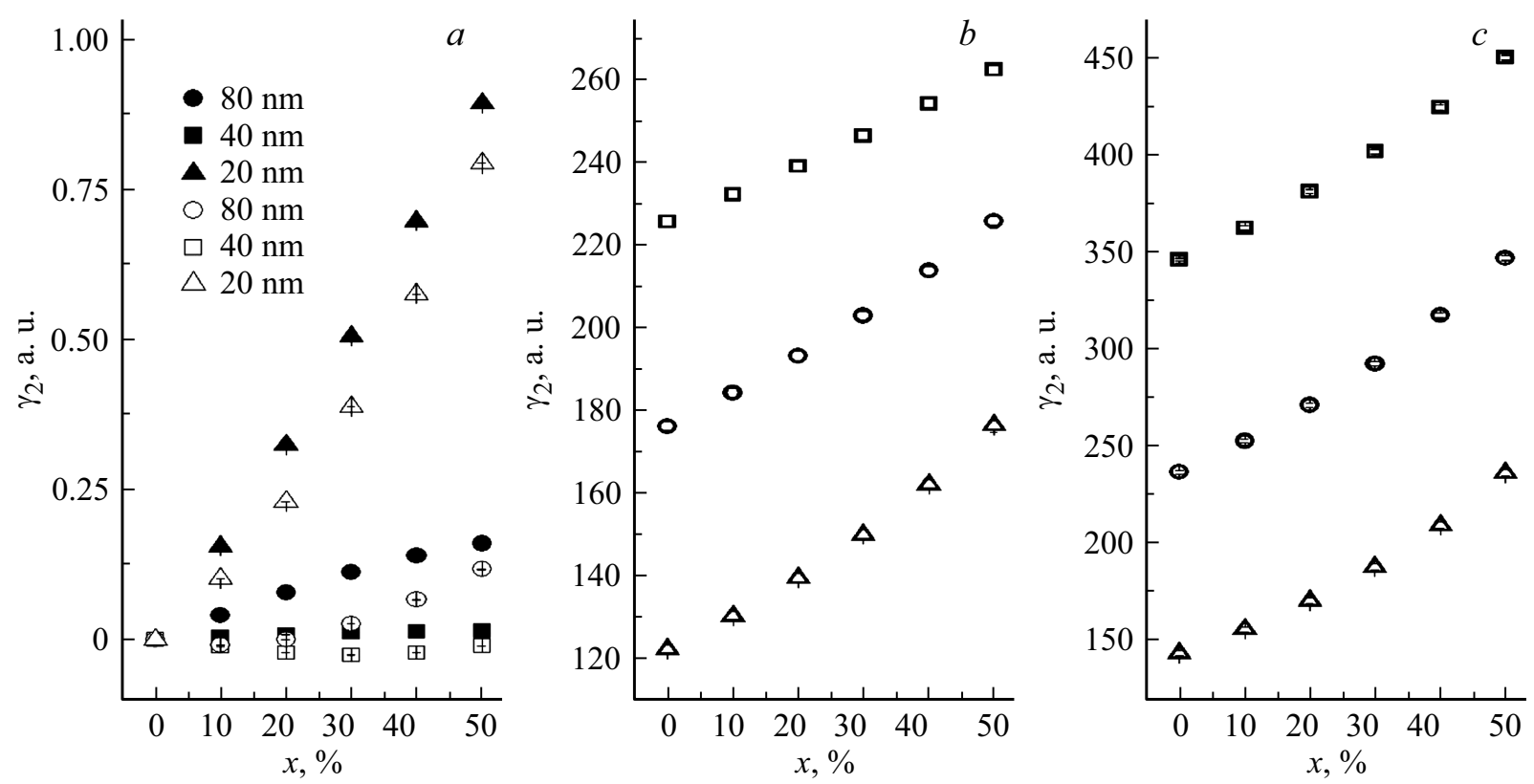

Рис. 2. Изменение коэффициента эксцесса $\left(\gamma_{2}\right)$ брэгговского рефлекса с увеличением весового процентного содержания фазы массивного материала в образце $(x)$ для разных функций профиля линии (гауссиан $(a)$, лоренциан $(b)$ и псевдо-войтиан $(c))$, размеров частиц и разного соотношения параметров решетки массива и наноструктурированной фазы (черные символы параметры решетки одинаковы, пустые символы - параметры решетки массива и наноструктурированной фазы различаются).
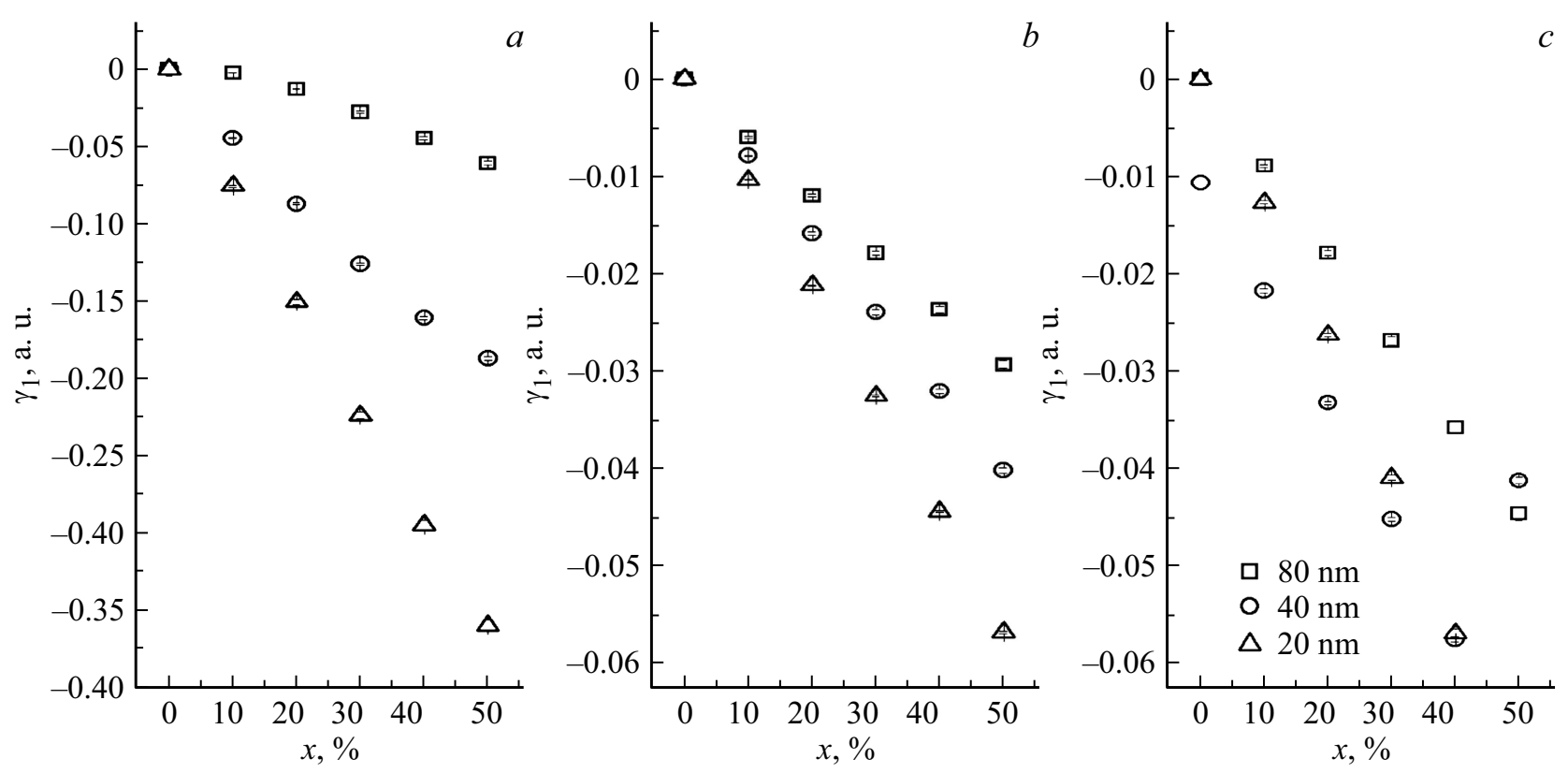

Рис. 3. Изменение коэффициента асимметрии $\left(\gamma_{1}\right)$ брэгговского рефлекса при увеличении весового процентного содержания массивного материала в образце $(x)$ для разных функций профиля линии (гауссиан $(a)$, лоренциан $(b)$ и псевдо-войтиан $(c))$ и размеров частиц.

с характерным размером менее $80 \mathrm{~nm}$ ) и около $5 \%$ для лоренциана и псевдо-войтиана для всех исследованных размеров наночастиц. Кроме того, как и на рис. 1, параметр $\gamma_{2}$ практически не зависит от разности параметров элементарной ячейки в случае лоренциана (рис. $2, b$ ) и псевдо-войтиана (рис. 2,c) 一 черные и белые символы совпадают.
Далее обратимся к поведению коэффициента асимметрии $\gamma_{1}$. Понятно, что соответствующие искажения формы линии в этом случае связаны с разностью параметров решетки $a$ для массивного и наноструктурированного материалов, поэтому на рис. 3 представлены только результаты расчетов для этого случая и для всех ранее упомянутых функций. Сразу отметим, что 

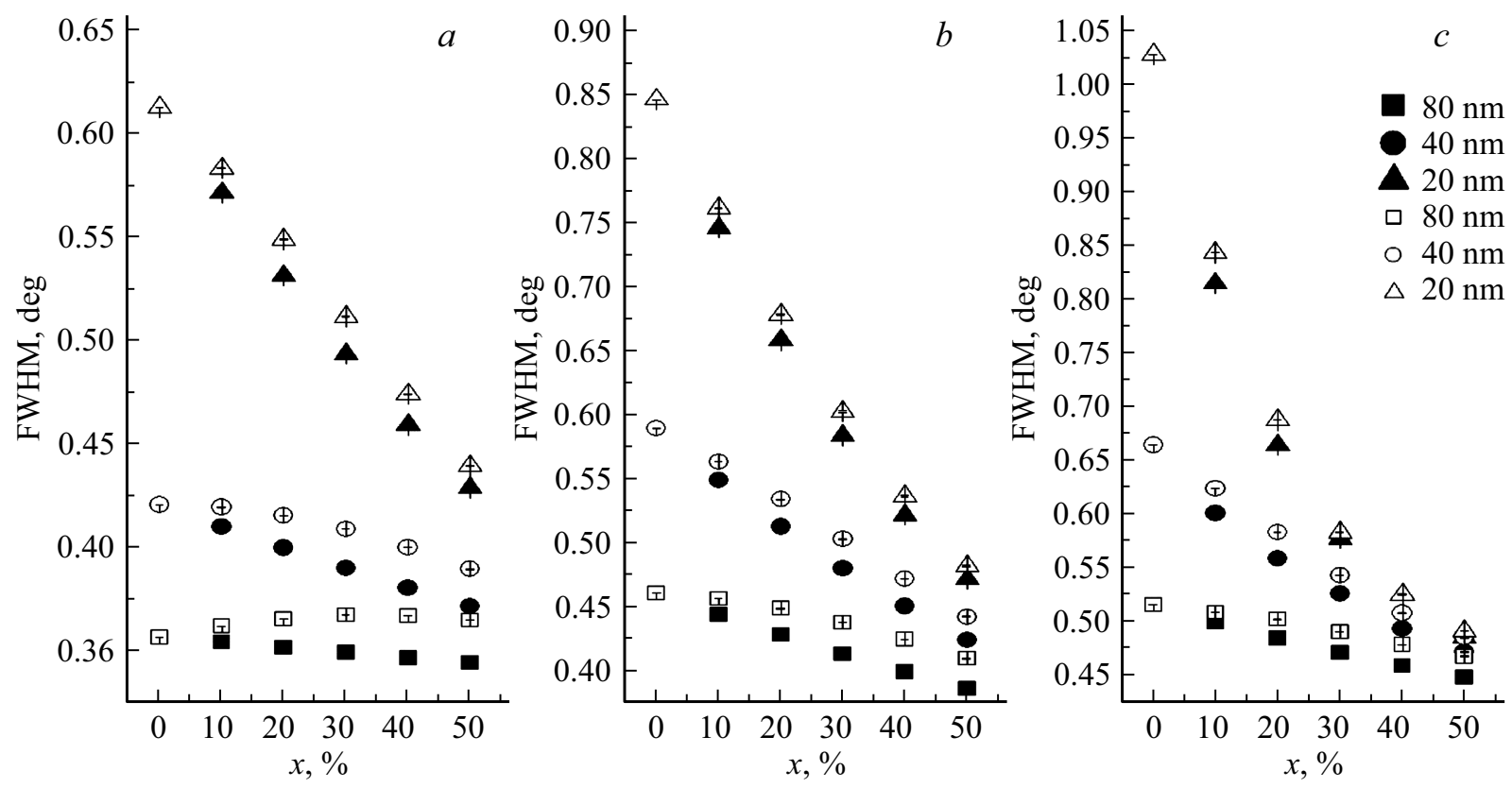

Рис. 4. Изменение ширины на полувысоте (FWHM) брэгговского рефлекса с увеличением весового процентного содержания фазы массивного материала в образце $(x)$ для разных функций профиля линии (гауссиан $(a)$, лоренциан $(b)$ и псевдо-войтиан $(c)$ ), размеров частиц и разного соотношения параметров решетки массива и наноструктурированной фазы (черные символы параметры решетки одинаковы, пустые символы - параметры решетки массива и наноструктурированной фазы различаются).

модуль коэффициента $\gamma_{1}$ при увеличении процентного содержания массивной фазы растет, т.е. чем больше размер частиц, тем $\gamma_{1}$ больше. При этом отчетливо видно, что для гауссиана (рис. 3,a) это изменение, т.е. разница между значениями $\gamma_{1}$ при разных концентрациях массива и разных дифракционных размерах наночастиц, существенно больше, чем для профильных функций Лоренца и Войта.

Из рис. 3, а можно видеть, что для НКМ, в котором, наряду с наночастицами с характерным размером 40 и $20 \mathrm{~nm}$, присутствует хотя бы 5\% примеси массивного материала, уже наблюдается вполне обнаружимая экспериментально асимметрия формы линии упругого пика. Что касается более крупных наночастиц $(80 \mathrm{~nm})$, то в этом случае уровень примеси массива в 5\% становится обнаружимым и для лоренциана и для псевдо-войтиана (рис. $3, b$ и $c$ ).

На последнем этапе, используя формулы (4), (5), (7) и (9), мы рассчитали зависимости полных ширин (в градусах) на полувысоте (FWHM) для всех рассмотренных ранее случаев (рис. 4) для нейтронного дифрактометра высокого разрешения FIREPOD E9.

Из представленных на рис. 4 зависимостей хорошо видно, что для малых наночастиц (с дифракционным размером менее $80 \mathrm{~nm}$ ) даже из анализа FWHM можно получить достаточно надежную оценку примеси массивного материала на уровне $5 \%$. Более того, оказывается возможным также оценить и разницу в параметре элементарной ячейки, если таковая присутствует. Таким образом, на основе изложенных выше рассуждений можно выстроить следующий алгоритм для предварительного тестирования (с использованием дифракции нейтронов или рентгеновского излучения) нанокомпозитных материалов с возможной примесью массивного материала:

1. Рассчитывается модельный спектр с учетом инструментального разрешения и для нескольких возможных (ожидаемых) дифракционных размеров наночастиц, введенных в поры использованной матрицы. Далее определяются FWHМ и моменты $\sigma, \gamma_{1}, \gamma_{2}$ данного распределения.

2. С хорошим разрешением проводятся измерения одного (в случае кубической структуры) интенсивного отражения при малых углах рассеяния пустой матрицы и массивного материала в области выбранного пика, а далее анализируются FWHМ и моменты $\sigma, \gamma_{1}, \gamma_{2}$ полученного углового распределения интенсивности для массивной фазы и для НКМ после вычитания фона от пустой матрицы. При этом необходимо обеспечить достаточную статистику, чтобы наблюдаемый эффект уширения спектра был за пределами ошибок определения FWHM.

3. Из результатов сравнения параметров модельных и экспериментального спектров проводятся оценки размеров наночастицы, ее параметров решетки и примеси массивного материала (как показано выше - в ряде случаев с точностью до 5 весовых процентов).

Полагаем, что предлагаемая процедура окажется весьма полезной при подготовке НКМ на основе молекулярных сит, так как не требует проведения полномасштабных дифракционных измерений и проведения 
полнопрофильного анализа полученных данных, не занимает много времени, доступна для применения даже на дифрактометрах с невысоким разрешением (необходимо только иметь точно определенную функцию разрешения прибора и хорошую статистическую обеспеченность данных) в случае наночастиц с малым дифракционным размером. Процедуру можно использовать и для аттестации НКМ на основе других пористых матриц, в которых возможно формирование крупных агломератов в трещинах, полостях и в других подобных дефектах матриц.

\section{Заключение}

В работе рассмотрен метод, позволяющий из анализа параметров одного дифракционного пика, полученного для нанокомпозитных материалов на основе нанопористых матриц, получить информацию о величине возможной примеси массивного материала в данном НКМ, оценить размеры наночастиц в НКМ и их параметры кристаллической структуры. В дальнейшем мы планируем рассмотреть и случаи с перекрестными функциями для описания инструментального разрешения и дифракционного пика от НКМ (например, гауссиан и лоренциан или гауссиан и псевдо-войтиан), а также и случаи, когда помимо массива и наноструктурированного материала в НКМ присутствует и примесь аморфной фазы.

\section{Финансирование работы}

О.А. Алексеева благодарит Российский фонд фундаментальных исследований (грант № 19-02-00760) за частичную финансовую поддержку при проведении исследований по данной тематике.

\section{Конфликт интересов}

Авторы заявляют, что у них нет конфликта интересов.

\section{Список литературы}

[1] А.А. Набережнов, Е.В. Стукова, О.А. Алексеева, C.A. Новикова, А. Franz. ЖТФ, 89(12), 1965 (2019). DOI: 10.21883/JTF.2019.12.48497.207-19 [A.A. Naberezhnov, E.V. Stukova, O.A. Alekseeva, S.A. Novikova, A. Franz. Tech. Phys., 64 (12), 1866 (2019). DOI: $10.1134 / \mathrm{S} 106378421912020 \mathrm{X}$

[2] G.K. Williamson, W.H. Hall. Acta Metallurgica, 1, 22 (1953). DOI: 10.1016/0001-6160(53)90006-6

[3] V.D. Mote, Y. Purushotham, B.N. Dole. J. Theor. Appl. Phys., 6, 6 (2012). DOI: 10.1186/2251-7235-6-6

[4] Y.T. Prabhu, K.V. Rao, V.S.S. Kumar, B.S. Kumari. World J. Nano Sci. Eng., 4, 21 (2014). DOI: $10.4236 /$ wjnse.2014.41004

[5] P. Thompson, D.E. Cox, J.B. Hastings. J. Appl. Cryst., 20, 79 (1987). DOI: $10.1107 / \mathrm{S} 0021889887087090$

[6] Г. Крамер. Математические методы статистики (Мир, M., 1975) 